\title{
Blocks of Monotone Boolean Functions
}

\author{
Tkachenco V.G. ${ }^{1, *}$, Sinyavsky O.V. ${ }^{2}$ \\ ${ }^{1}$ Odessa National Academy of Telecommunications named after O. S. Popov, Ukraine \\ ${ }^{2}$ Military Academy (Odessa), Ukraine
}

Copyright $\bigcirc 2016$ by authors, all rights reserved. Authors agree that this article remains permanently open access under the terms of the Creative Commons Attribution License 4.0 International License

\begin{abstract}
This paper first proposed the method of constructing blocks of monotone Boolean functions (MBFs) is developed for classification and the analysis of these functions. Use of only nonisomorphic blocks considerably simplifies enumeration MBFs. Application of the method of construction blocks on the example of classification and analysis of MBFs from 0 to 4 variables is considered. This method can be used at counting of all MBFs of a given rank $n$.
\end{abstract}

Keywords Monotone Boolean Functions, Disjunctive Complement, Conjunctive Complement, Dedekind Number, Free Distributive Lattice

\section{Introduction}

In 1897 R. Dedekind has published article [1] in which the number of elements of a free distributive lattice with four generators has been found. The number $\psi(n)$ of elements of a free distributive lattice with $\mathrm{n}$ generators coincides with number of anti-chains in the unit n-dimensional cube. In the language of logic algebra $K(n)=\psi(n)+2$ - the number of the monotone Boolean functions (MBFs), which depend on $n$ variables $x_{1}, \ldots, x_{n}$. The problem of calculating $\psi(n)$ is called Dedekind's problem. $D(0)-D(4)$ computed by R. Dedekind (1897). $D(5)$ are given by Church (1940). $D(6)$ was calculated by Ward (1946), $D(7)$ was calculated by Church (1965) and $D(8)$ by Wiedemann [2] (1991). As it turned out, this problem is quite difficult and cannot be examine through the traditional method of generating functions. At present known ways of calculation $D(n)$. One way of partitioning the number of distinct monotone functions of $n$ variables is to classify them according to the number of distinct input states, at which the function is equal to 1 . Another way of splitting up $D(n)$ is according with the number of conjunctions in the disjunctive normal form.[3]

In $[4,5]$ developed a classification of the types of MBFs and enumeration of maximum types of MBFs. In [6] proved the expression for enumeration of types MBFs as a product of matrices.
However, the literature is not considered a method of analysis and classification of MBFs - based structure MBFs blocks (sets of MBFs connected by three operations considered further), which in some cases can reduce sorting MBFs due to that we can to exclude all isomorphic blocks and consider only nonisomorphic blocks. The analysis of MBFs blocks allows examining this problem in a new way.

This paper first proposed the classification method and research MBFs with the help of the blocks. This method is not limited to the number of variables, because for any MBF with any number of variables $\mathrm{n}$ using the disjunctive complement s and duality can construct a block containing this MBF. For descriptions of all MBFs of $n$ variables it is sufficient to construct only nonisomorphic blocks. This very significantly reduces the description of all MBFs of $n$ variables. For example, all MBFs of 5 variables (these MBFs blocks will be described in the following paper) can be divided into 522 blocks, but you can only choose 23 pairwise nonisomorphic. Nonisomorphic blocks are directly related to Dedekind numbers.

The aim of paper is development of a method of the analysis and MBFs classification on the basis of construction of MBFs blocks.

\section{Results}

Let's remind the main concepts connected with MBFs. Boolean function $f\left(x_{1}, \ldots, x_{n}\right)$ is called as monotone, if for any pairs of sets of values of variables $\left(a_{1}, \ldots, a_{n}\right)$ and $\left(b_{1}, \ldots, b_{n}\right)$ for which the relation $\left(a_{1}, \ldots, a_{n}\right) \leq\left(b_{1}, \ldots, b_{n}\right)$ truly and an inequality is carried out $f\left(a_{1}, \ldots, a_{n}\right) \leq f\left(b_{1}, \ldots, b_{n}\right)$. For a disjunctive normal form of MBFs it means that in it there is no negation operation, and there are only conjunction and disjunction operations.

The vector $P=\left(a_{n}, \ldots, a_{i}, \ldots, a_{1}, a_{0}\right)$, the components of which take values from the set $\{0,1\}$ is called [5] input set of Boolean function of $n$ variables. The set of all input sets forms Boolean cube of rank $n$. themselves input sets $\mathrm{P}$ are tops of the Boolean cube. Any Boolean function is defined by a set of vertices of the Boolean cube, in which the 
function is equal to unity. Any set of incomparable tops of a Boolean cube is called as an antichain to set the MBFs [5] is sufficient to indicate some antichain in a Boolean cube. Each top of an antichain (except tops corresponding to entrance sets $(0 \ldots, 0)$ and $(1 \ldots, 1))$ defines conjunction in a disjunctive normal form of MBFs corresponding to this antichain.

Consider one of the ways to describe the MBFs as a minimum input sets or the corresponding family of subsets Sperner. (Any family of subsets of a set is called a family of subsets Sperner, if none of the subsets of the family is not contained in any other subset of the same family.) In this case, if MBFs of $n$ variables, then an arbitrary subset of a family of subsets Sperner may contain from 0 to $n$ elements.

Let's consider all MBFs of ranks from 0 to 4 . There are only two MBFs of rank 0 . It is $f_{0}(0)$ identically equal 0 and $f_{1}(0)$ identically equal 1 . There are three MBFs of rank 1 . It is $f_{0}(1)$ identically equal $0, f_{1}(1)$ identically equal 1 and $f_{2}(1)=x_{1}$. There are six MBFs of rank 2. It is $f_{0}(2)$ identically equal 0 , $f_{1}(2)$ identically equal $1, f_{2}(2)=x_{1} x_{2}, f_{3}(2)=x_{1} \vee x_{2}, f_{4}(2)=x_{1}$, $f_{5}(2)=x_{2}(\vee$-disjunction operations). There are twenty MBFs of a rank 3 . It is $f_{0}(3)$ identically equal $0 ; f_{1}(3)$ identically equal $1 ; f_{2}(3)=x_{1} x_{2} x_{3} ; f_{3}(3)=x_{1} \vee x_{2} \vee x_{3} ; f_{4}(3)=$ $x_{1} x_{2} \vee x_{1} x_{3} \vee x_{2} x_{3} ; f_{5}(3)=x_{1} ; f_{6}(3)=x_{2} x_{3} ; f_{7}(3)=x_{2} \vee x_{3} ; f_{8}(3)=$ $x_{1} x_{2} \vee x_{1} x_{3} ; f_{9}(3)=x_{1} \vee x_{2} x_{3} ; f_{10}(3)=x_{2} ; f_{11}(3)=x_{1} x_{3} ; f_{12}(3)=$ $x_{1} \vee x_{3} ; f_{13}(3)=x_{1} x_{2} \vee x_{2} x_{3} ; f_{14}(3)=x_{2} \vee x_{1} x_{3} ; f_{15}(3)=x_{3} ; f_{16}(3)$ $=x_{1} x_{2} ; f_{17}(3)=x_{1} \vee x_{2} ; f_{18}(3)=x_{1} x_{3} \vee x_{2} x_{3}$ and $f_{19}(3)=x_{3} \vee x_{1} x_{2}$.

In the following table all 168 MBFs of rank 4 are given in the minimal disjunctive form. MBFs with number 0 , that is $f_{0}(4)$ is zero MBFs (it is equal 0 at all values of entrance variables), and $f_{1}(4)$ - unit MBFs.

\begin{tabular}{|c|c|c|c|c|c|}
\hline $\begin{array}{c}\text { № } \\
\text { MBFs }\end{array}$ & Disjunctive normal form & $\begin{array}{c}\text { № } \\
\text { MBFs }\end{array}$ & Disjunctive normal form & $\begin{array}{c}\text { № } \\
\text { MBFs }\end{array}$ & Disjunctive normal form \\
\hline 0 & 0 conjunctions & 56 & $x_{1} x_{2} \vee x_{1} x_{4} \vee x_{2} x_{3}$ & 112 & $x_{1} \vee x_{2} x_{3} \vee x_{3} x_{4}$ \\
\hline 1 & is not present(unit MBFs) & 57 & $x_{1} x_{4} \vee x_{2} x_{3} \vee x_{3} x_{4}$ & 113 & $x_{1} x_{2} \vee x_{1} x_{4} \vee x_{2} x_{3} x_{4}$ \\
\hline 2 & $x_{1} x_{2} x_{3} x_{4}$ & 58 & $x_{1} x_{3} \vee x_{2} x_{4} \vee x_{3} x_{4}$ & 114 & $x_{1} x_{2} \vee x_{1} x_{3} \vee x_{1} x_{4} \vee x_{2} x_{4}$ \\
\hline 3 & $x_{1} \vee x_{2} \vee x_{3} \vee x_{4}$ & 59 & $x_{1} x_{2} \vee x_{1} x_{3} \vee x_{2} x_{4}$ & 115 & $x_{1} x_{3} \vee x_{2} x_{3} \vee x_{2} x_{4} \vee x_{3} x_{4}$ \\
\hline 4 & $x_{1} x_{2} x_{3} \vee x_{1} x_{2} x_{4} \vee x_{1} x_{3} x_{4} \vee x_{2} x_{3} x_{4}$ & 60 & $x_{1} x_{2}$ & 116 & $x_{2} x_{3} \vee x_{3} x_{4} \vee x_{1} x_{2} x_{4}$ \\
\hline 5 & $x_{1} x_{2} \vee x_{1} x_{3} \vee x_{1} x_{4} \vee x_{2} x_{3} \vee x_{2} x_{4} \vee x_{3} x_{4}$ & 61 & $x_{3} x_{4}$ & 117 & $x_{3} \vee x_{1} x_{2} \vee x_{1} x_{4}$ \\
\hline 6 & $x_{1}$ & 62 & $x_{3} \vee x_{4}$ & 118 & $x_{1} x_{3} \vee x_{2} x_{3} x_{4}$ \\
\hline 7 & $x_{2} x_{3} x_{4}$ & 63 & $x_{1} x_{2} x_{3} \vee x_{1} x_{2} x_{4}$ & 119 & $x_{1} \vee x_{2} x_{4}$ \\
\hline 8 & $x_{2} \vee x_{3} \vee x_{4}$ & 64 & $x_{1} \vee x_{2} \vee x_{3} x_{4}$ & 120 & $x_{1} x_{2} \vee x_{2} x_{3}$ \\
\hline 9 & $x_{1} x_{2} x_{3} \vee x_{1} x_{2} x_{4} \vee x_{1} x_{3} x_{4}$ & 65 & $x_{1} x_{2} \vee x_{1} x_{3} x_{4} \vee x_{2} x_{3} x_{4}$ & 121 & $x_{1} x_{4} \vee x_{3} x_{4}$ \\
\hline 10 & $x_{1} \vee x_{2} x_{3} \vee x_{2} x_{4} \vee x_{3} x_{4}$ & 66 & $x_{1} x_{2} \vee x_{1} x_{3} \vee x_{1} x_{4} \vee x_{2} x_{3} \vee x_{2} x_{4}$ & 122 & $x_{4} \vee x_{1} x_{3}$ \\
\hline 11 & $x_{1} x_{2} \vee x_{1} x_{3} \vee x_{1} x_{4} \vee x_{2} x_{3} x_{4}$ & 67 & $x_{1} x_{3} \vee x_{1} x_{4} \vee x_{2} x_{3} \vee x_{2} x_{4} \vee x_{3} x_{4}$ & 123 & $x_{2} x_{4} \vee x_{1} x_{2} x_{3}$ \\
\hline 12 & $x_{2}$ & 68 & $x_{3} x_{4} \vee x_{1} x_{2} x_{3} \vee x_{1} x_{2} x_{4}$ & 124 & $x_{2} \vee x_{1} x_{4} \vee x_{3} x_{4}$ \\
\hline 13 & $x_{1} x_{3} x_{4}$ & 69 & $x_{3} \vee x_{4} \vee x_{1} x_{2}$ & 125 & $x_{1} x_{2} \vee x_{2} x_{3} \vee x_{1} x_{3} x_{4}$ \\
\hline 14 & $x_{1} \vee x_{3} \vee x_{4}$ & 70 & $x_{1} x_{3} x_{4} \vee x_{2} x_{3} x_{4}$ & 126 & $x_{1} x_{2} \vee x_{1} x_{3} \vee x_{2} x_{3} \vee x_{2} x_{4}$ \\
\hline 15 & $x_{1} x_{2} x_{3} \vee x_{1} x_{2} x_{4} \vee x_{2} x_{3} x_{4}$ & 71 & $x_{1} \vee x_{2}$ & 127 & $x_{1} x_{3} \vee x_{1} x_{4} \vee x_{2} x_{4} \vee x_{3} x_{4}$ \\
\hline 16 & $x_{2} \vee x_{1} x_{3} \vee x_{1} x_{4} \vee x_{3} x_{4}$ & 72 & $x_{1} x_{3}$ & 128 & $x_{1} x_{4} \vee x_{3} x_{4} \vee x_{1} x_{2} x_{3}$ \\
\hline 17 & $x_{1} x_{2} \vee x_{2} x_{3} \vee x_{2} x_{4} \vee x_{1} x_{3} x_{4}$ & 73 & $x_{2} x_{4}$ & 129 & $x_{4} \vee x_{1} x_{2} \vee x_{2} x_{3}$ \\
\hline 18 & $x_{3}$ & 74 & $x_{2} \vee x_{4}$ & 130 & $x_{2} x_{4} \vee x_{1} x_{3} x_{4}$ \\
\hline 19 & $x_{1} x_{2} x_{4}$ & 75 & $x_{1} x_{2} x_{3} \vee x_{1} x_{3} x_{4}$ & 131 & $x_{2} \vee x_{1} x_{3}$ \\
\hline 20 & $x_{1} \vee x_{2} \vee x_{4}$ & 76 & $x_{1} \vee x_{3} \vee x_{2} x_{4}$ & 132 & $x_{1} x_{2} \vee x_{2} x_{4}$ \\
\hline 21 & $x_{1} x_{2} x_{3} \vee x_{1} x_{3} x_{4} \vee x_{2} x_{3} x_{4}$ & 77 & $x_{1} x_{3} \vee x_{1} x_{2} x_{4} \vee x_{2} x_{3} x_{4}$ & 133 & $x_{1} x_{3} \vee x_{3} x_{4}$ \\
\hline 22 & $x_{3} \vee x_{1} x_{2} \vee x_{1} x_{4} \vee x_{2} x_{4}$ & 78 & $x_{1} x_{2} \vee x_{1} x_{3} \vee x_{1} x_{4} \vee x_{2} x_{3} \vee x_{3} x_{4}$ & 134 & $x_{3} \vee x_{1} x_{4}$ \\
\hline 23 & $x_{1} x_{3} \vee x_{2} x_{3} \vee x_{3} x_{4} \vee x_{1} x_{2} x_{4}$ & 79 & $x_{1} x_{2} \vee x_{1} x_{4} \vee x_{2} x_{3} \vee x_{2} x_{4} \vee x_{3} x_{4}$ & 135 & $x_{2} x_{3} \vee x_{1} x_{2} x_{4}$ \\
\hline 24 & $x_{4}$ & 80 & $x_{2} x_{4} \vee x_{1} x_{2} x_{3} \vee x_{1} x_{3} x_{4}$ & 136 & $x_{2} \vee x_{1} x_{3} \vee x_{3} x_{4}$ \\
\hline 25 & $x_{1} x_{2} x_{3}$ & 81 & $x_{2} \vee x_{4} \vee x_{1} x_{3}$ & 137 & $x_{1} x_{2} \vee x_{2} x_{4} \vee x_{1} x_{3} x_{4}$ \\
\hline 26 & $x_{1} \vee x_{2} \vee x_{3}$ & 82 & $x_{1} x_{2} x_{4} \vee x_{2} x_{3} x_{4}$ & 138 & $x_{1} x_{2} \vee x_{1} x_{4} \vee x_{2} x_{3} \vee x_{2} x_{4}$ \\
\hline 27 & $x_{1} x_{2} x_{4} \vee x_{1} x_{3} x_{4} \vee x_{2} x_{3} x_{4}$ & 83 & $x_{1} \vee x_{3}$ & 139 & $x_{1} x_{3} \vee x_{1} x_{4} \vee x_{2} x_{3} \vee x_{3} x_{4}$ \\
\hline 28 & $x_{4} \vee x_{1} x_{2} \vee x_{1} x_{3} \vee x_{2} x_{3}$ & 84 & $x_{1} x_{4}$ & 140 & $x_{1} x_{3} \vee x_{3} x_{4} \vee x_{1} x_{2} x_{4}$ \\
\hline 29 & $x_{1} x_{4} \vee x_{2} x_{4} \vee x_{3} x_{4} \vee x_{1} x_{2} x_{3}$ & 85 & $x_{2} x_{3}$ & 141 & $x_{3} \vee x_{1} x_{2} \vee x_{2} x_{4}$ \\
\hline 30 & $x_{1} x_{2} \vee x_{3} x_{4}$ & 86 & $x_{2} \vee x_{3}$ & 142 & $x_{2} x_{3} \vee x_{1} x_{3} x_{4}$ \\
\hline 31 & $x_{1} x_{3} \vee x_{1} x_{4} \vee x_{2} x_{3} \vee x_{2} x_{4}$ & 87 & $x_{1} x_{2} x_{4} \vee x_{1} x_{3} x_{4}$ & 143 & $x_{2} \vee x_{1} x_{4}$ \\
\hline 32 & $x_{1} x_{3} \vee x_{2} x_{4}$ & 88 & $x_{1} \vee x_{4} \vee x_{2} x_{3}$ & 144 & $x_{1} x_{3} \vee x_{1} x_{4}$ \\
\hline 33 & $x_{1} x_{2} \vee x_{1} x_{4} \vee x_{2} x_{3} \vee x_{3} x_{4}$ & 89 & $x_{1} x_{4} \vee x_{1} x_{3} x_{4} \vee x_{2} x_{3} x_{4}$ & 145 & $x_{2} x_{3} \vee x_{2} x_{4}$ \\
\hline 34 & $x_{1} x_{4} \vee x_{2} x_{3}$ & 90 & $x_{1} x_{2} \vee x_{1} x_{3} \vee x_{1} x_{4} \vee x_{2} x_{4} \vee x_{3} x_{4}$ & 146 & $x_{2} \vee x_{3} x_{4}$ \\
\hline
\end{tabular}




\begin{tabular}{|c|c|c|c|c|c|}
\hline 35 & $x_{1} x_{2} \vee x_{1} x_{3} \vee x_{2} x_{4} \vee x_{3} x_{4}$ & 91 & $x_{1} x_{2} \vee x_{1} x_{3} \vee x_{2} x_{3} \vee x_{2} x_{4} \vee x_{3} x_{4}$ & 147 & $x_{1} x_{2} \vee x_{1} x_{3} x_{4}$ \\
\hline 36 & $x_{2} x_{3} \vee x_{2} x_{4} \vee x_{3} x_{4}$ & 92 & $x_{2} x_{3} \vee x_{1} x_{2} x_{4} \vee x_{1} x_{3} x_{4}$ & 148 & $x_{1} \vee x_{2} x_{3} \vee x_{2} x_{4}$ \\
\hline 37 & $x_{1} x_{2} \vee x_{1} x_{3} \vee x_{1} x_{4}$ & 93 & $x_{2} \vee x_{3} \vee x_{1} x_{4}$ & 149 & $x_{1} x_{3} \vee x_{1} x_{4} \vee x_{2} x_{3} x_{4}$ \\
\hline 38 & $x_{1} \vee x_{2} x_{3} x_{4}$ & 94 & $x_{1} x_{2} x_{3} \vee x_{2} x_{3} x_{4}$ & 150 & $x_{1} x_{2} \vee x_{1} x_{3} \vee x_{1} x_{4} \vee x_{3} x_{4}$ \\
\hline 39 & $x_{1} x_{3} \vee x_{1} x_{4} \vee x_{3} x_{4}$ & 95 & $x_{1} \vee x_{4}$ & 151 & $x_{1} x_{2} \vee x_{2} x_{3} \vee x_{2} x_{4} \vee x_{3} x_{4}$ \\
\hline 40 & $x_{1} x_{2} \vee x_{2} x_{3} \vee x_{2} x_{4}$ & 96 & $x_{1} x_{2} \vee x_{1} x_{3}$ & 152 & $x_{2} x_{3} \vee x_{2} x_{4} \vee x_{1} x_{3} x_{4}$ \\
\hline 41 & $x_{2} \vee x_{1} x_{3} x_{4}$ & 97 & $x_{2} x_{4} \vee x_{3} x_{4}$ & 153 & $x_{2} \vee x_{1} x_{3} \vee x_{1} x_{4}$ \\
\hline 42 & $x_{1} x_{2} \vee x_{1} x_{4} \vee x_{2} x_{4}$ & 98 & $x_{4} \vee x_{2} x_{3}$ & 154 & $x_{1} x_{2} \vee x_{2} x_{3} x_{4}$ \\
\hline 43 & $x_{1} x_{3} \vee x_{2} x_{3} \vee x_{3} x_{4}$ & 99 & $x_{1} x_{4} \vee x_{1} x_{2} x_{3}$ & 155 & $x_{1} \vee x_{3} x_{4}$ \\
\hline 44 & $x_{3} \vee x_{1} x_{2} x_{4}$ & 100 & $x_{1} \vee x_{2} x_{4} \vee x_{3} x_{4}$ & 156 & $x_{1} x_{3} \vee x_{2} x_{3}$ \\
\hline 45 & $x_{1} x_{2} \vee x_{1} x_{3} \vee x_{2} x_{3}$ & 101 & $x_{1} x_{2} \vee x_{1} x_{3} \vee x_{2} x_{3} x_{4}$ & 157 & $x_{1} x_{4} \vee x_{2} x_{4}$ \\
\hline 46 & $x_{1} x_{4} \vee x_{2} x_{4} \vee x_{3} x_{4}$ & 102 & $x_{1} x_{2} \vee x_{1} x_{3} \vee x_{1} x_{4} \vee x_{2} x_{3}$ & 158 & $x_{4} \vee x_{1} x_{2}$ \\
\hline 47 & $x_{4} \vee x_{1} x_{2} x_{3}$ & 103 & $x_{1} x_{4} \vee x_{2} x_{3} \vee x_{2} x_{4} \vee x_{3} x_{4}$ & 159 & $x_{3} x_{4} \vee x_{1} x_{2} x_{3}$ \\
\hline 48 & $x_{1} x_{2} \vee x_{2} x_{3} \vee x_{3} x_{4}$ & 104 & $x_{2} x_{4} \vee x_{3} x_{4} \vee x_{1} x_{2} x_{3}$ & 160 & $x_{3} \vee x_{1} x_{4} \vee x_{2} x_{4}$ \\
\hline 49 & $x_{1} x_{2} \vee x_{1} x_{4} \vee x_{3} x_{4}$ & 105 & $x_{4} \vee x_{1} x_{2} \vee x_{1} x_{3}$ & 161 & $x_{1} x_{3} \vee x_{2} x_{3} \vee x_{1} x_{2} x_{4}$ \\
\hline 50 & $x_{1} x_{3} \vee x_{1} x_{4} \vee x_{2} x_{4}$ & 106 & $x_{1} x_{4} \vee x_{2} x_{3} x_{4}$ & 162 & $x_{1} x_{2} \vee x_{1} x_{3} \vee x_{2} x_{3} \vee x_{3} x_{4}$ \\
\hline 51 & $x_{1} x_{3} \vee x_{2} x_{3} \vee x_{2} x_{4}$ & 107 & $x_{1} \vee x_{2} x_{3}$ & 163 & $x_{1} x_{2} \vee x_{1} x_{4} \vee x_{2} x_{4} \vee x_{3} x_{4}$ \\
\hline 52 & $x_{1} x_{2} \vee x_{1} x_{3} \vee x_{3} x_{4}$ & 108 & $x_{1} x_{2} \vee x_{1} x_{4}$ & 164 & $x_{1} x_{4} \vee x_{2} x_{4} \vee x_{1} x_{2} x_{3}$ \\
\hline 53 & $x_{1} x_{2} \vee x_{2} x_{4} \vee x_{3} x_{4}$ & 109 & $x_{2} x_{3} \vee x_{3} x_{4}$ & 165 & $x_{4} \vee x_{1} x_{3} \vee x_{2} x_{3}$ \\
\hline 54 & $x_{1} x_{4} \vee x_{2} x_{3} \vee x_{2} x_{4}$ & 110 & $x_{3} \vee x_{2} x_{4}$ & 166 & $x_{3} x_{4} \vee x_{1} x_{2} x_{4}$ \\
\hline 55 & $x_{1} x_{3} \vee x_{1} x_{4} \vee x_{2} x_{3}$ & 111 & $x_{1} x_{3} \vee x_{1} x_{2} x_{4}$ & 167 & $x_{3} \vee x_{1} x_{2}$ \\
\hline
\end{tabular}

All MBFs of one rank form a distributive lattice with respect to the operations of conjunction and disjunction. Such lattices of $R_{0}, R_{1}, R_{2}$ and $R_{3}$ for MBFs ranks from 0 to 3 are represented on fig. 1. Lattices of $R_{1}, R_{2}$ and $R_{3}$ differ from free distributive lattices of the same rank complement of the highest and lowest tops.

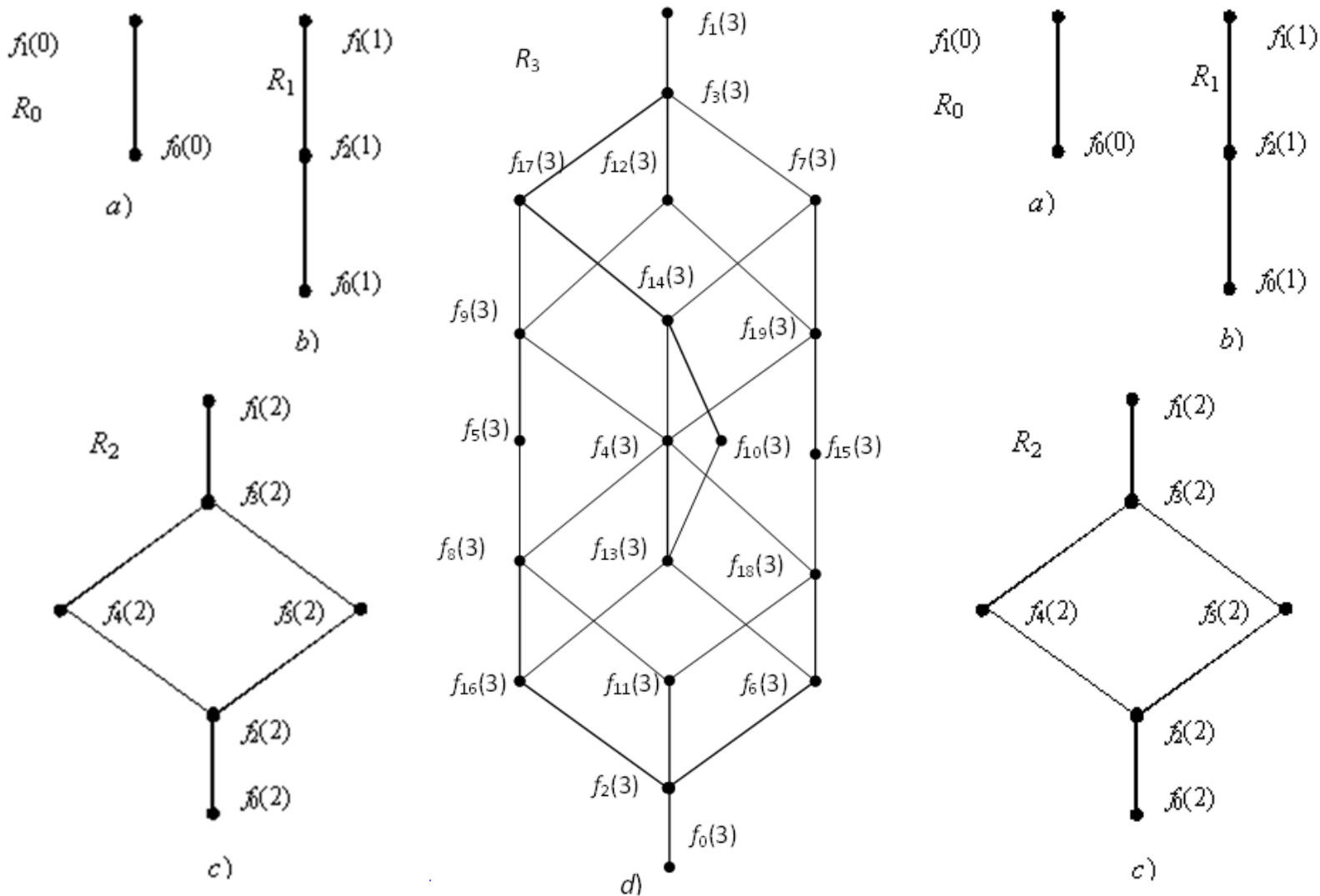

Figure 1. Lattices MBFs $a$ ) $\left.\left.R_{0}, b\right) R_{l}, c\right) R_{2}$ and $d$ ) $R_{3}$ 


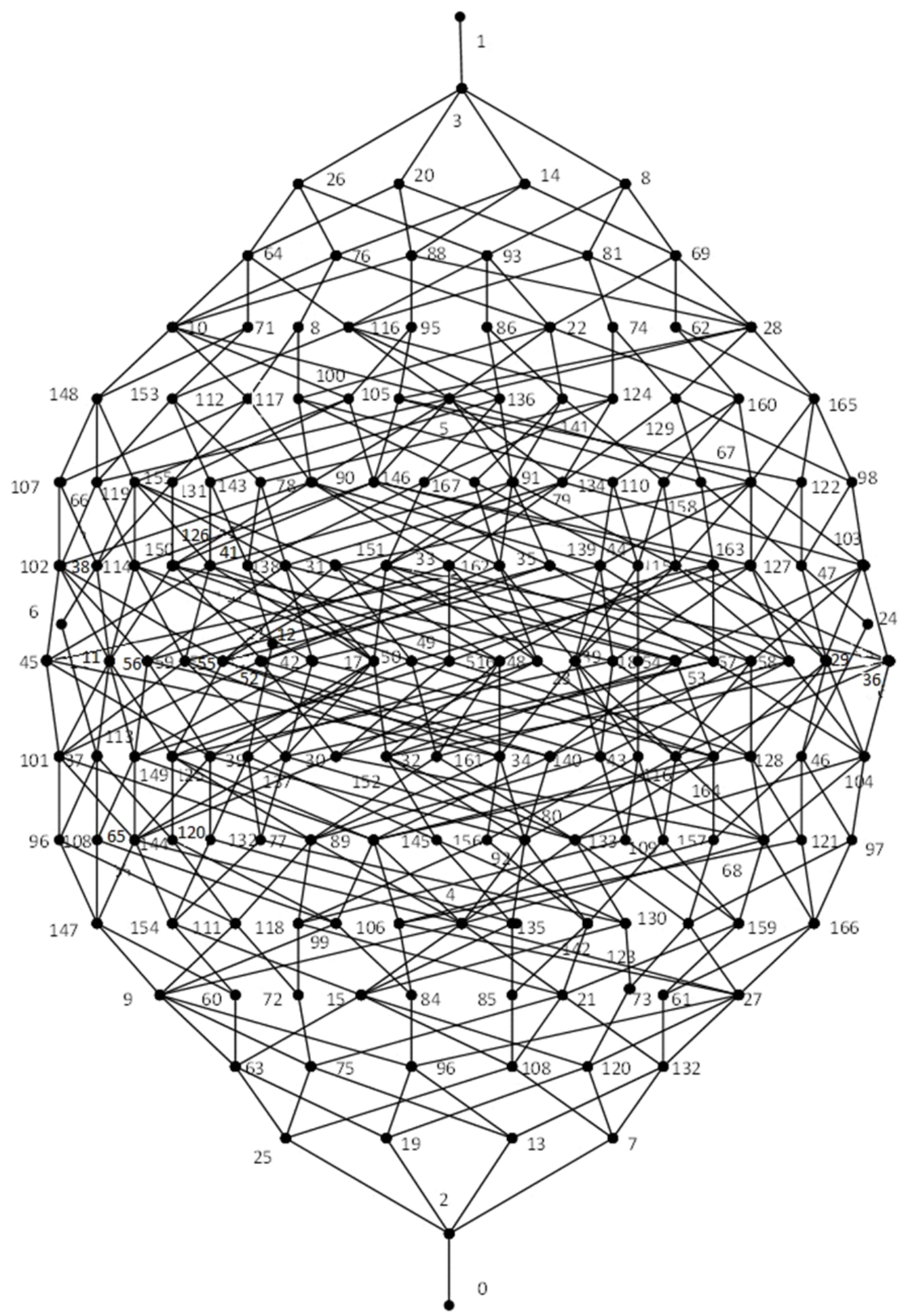

Figure 2. The Lattice of MBFs 4 ranks

On fig. 2 the lattice of all MBFs of rank 4 is shown. Numbers MBFs coincide with the numbers in the table above. If discard MBFs $f_{0}(4)$ and $f_{1}(4)$, the lattice in Fig. 2 is a free distributive lattice of rank 4.

In [7] on the set of MBFs any rank identified three unary operations: duality, conjunctive complement and disjunctive complement. For disjunctive complement $\overline{f_{i}}(n)$ from the $i$-th MBFs $f_{\mathrm{i}}(n)$ must be replaced in minimal disjunctive form each conjunction of $m$ variables on the conjunction of all $n$ $m$ variables not included in the initial conjunction. For conjunctive complement $\underline{f_{i}}(n)$ from the $i$-th MBFs $f_{i}(n)$ must be replaced in minimal conjunctive form of each disjunction of $\mathrm{m}$ variables on the disjunction of all $n-m$ variables not included in the initial disjunction. For dual MBFs $f i^{-1}(n)$ from the $i$-th MBFs $f_{i}(n)$ must be replaced in minimal disjunctive form all the operations of conjunction 
with disjunction operations and simultaneously replace all operations disjunction operations with conjunction operations. In this dual MBFs $f_{i}^{-1}(\mathrm{n})$ is obtained in the minimal conjunctive form. For dual MBFs $f_{i}^{-1}(n)$ in the minimal disjunctive form should be received in the form of minimum conjunctive open the brackets and cause similar terms.

Block MBFs rank $\mathrm{n}$ is a subset of all MBFs rank $\mathrm{n}$, closed with respect to the three operations: duality, conjunctive complement and disjunctive complement. We introduce some definitions. Block power is the number of MBFs which enter into it. Two blocks are similar, if the same power and abstraction from their member of MBFs, these blocks are indistinguishable. Two blocks are isomorphic if any MBFs one block can be obtained from some other MBFs block certain substitution variables. In complement, if both of the MBFs to perform one of three operations defined for the block, then the resulting MBFs first block is obtained from the resultant MBFs another unit of the same substitution variables. By definition isomorphic blocks are similar.

MBFs of ranks 0 and 1 are grouped in one block consisting and two and of three MBFs respectively with respect to considered three operations. MBFs rank 2 may be represented as two blocks, one of which consists of four MBFs, and another - of the two MBFs. All these blocks are shown in Fig. 3. Here, the opera tion of duality is represented by the solid line, the operation of disjunctive complement - a dashed line and the operation of conjunctive complement dash-dotted line. For example, in a unit consisting of MBFs $f_{0}(2), f_{1}(2), f_{2}(2)$ and $f_{3}(2)$ we have: $f_{1}(2)=f_{0}^{-1}(2), f_{3}(2)=\underline{f_{0}}(2)$ and $f_{2}(2)=\overline{f_{1}}(2)$.

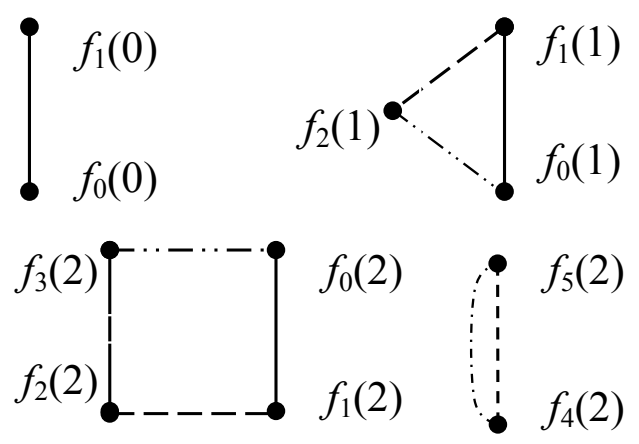

Figure 3. MBFs Blocks of ranks from 0 to 2

All MBFs of rank 3 split into 4 blocks of 5 elements. On Fig. 4 shows these blocks. MBFs $f_{5}(3), f_{10}(3), f_{15}(3)$ an $f_{4}(3)$ are self-dual.

All MBFs of any of blocks on fig. 4 can be obtained from one MBFs, applying to it above all at first operation of disjunctive complement, and then duality operation. So, for example, the second block in the top row is generated from MBFs $f_{5}(3)=x_{1}$ by receiving a chain of MBFs: $f_{5}(3), f_{6}(3)$, $f_{7}(3), f_{8}(3), f_{9}(3)$. It is similarly possible to receive a chain of $f_{10}(3), f_{11}(3), f_{12}(3), f_{13}(3), f_{14}(3)$, a chain of $f_{15}(3), f_{16}(3), f_{17}(3)$, $f_{18}(3), f_{19}(3)$ and a chain of $f_{4}(3), f_{3}(3), f_{2}(3), f_{1}(3), f_{0}(3)$ of $\operatorname{MBFs} f_{10}(3)=x_{2}$, MBFs $f_{15}(3)=x_{3}$ and MBFs $f_{4}(3)=x_{1} x_{2} \vee$ $x_{1} x_{3} \vee x_{2} x_{3}$ respectively. These blocks, as shown in Fig. 4, i.e. with the same number and the same form MBFs said to be similar.

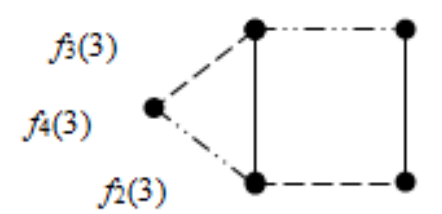

fo(3)
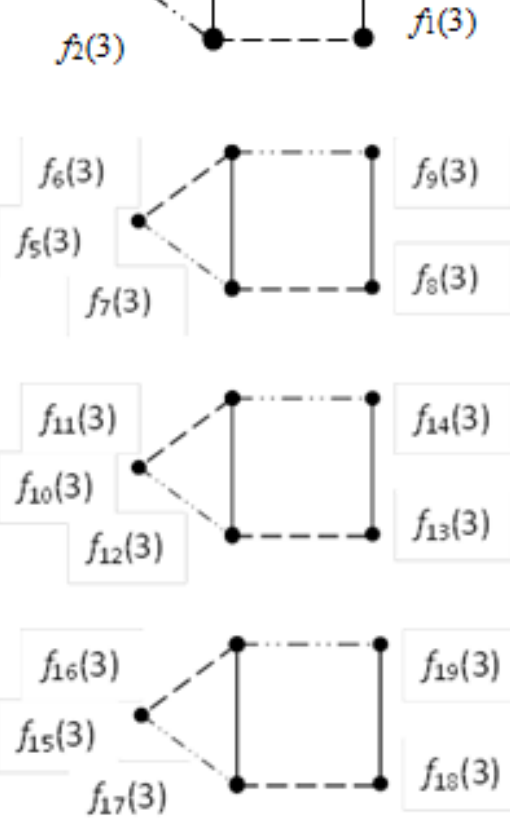

Figure 4. MBFs Blocks of rank 3

On Fig. 5 shows three block MBFs of rank of 4 power of 2 (a) Block 1), 3 (b) Block 2) and 4 (c) Block 3). Here, the operation of duality is represented by the solid line, the operation of disjunctive complement dashed line and the operation of conjunctive complement dash-dotted line.



Figure 5. MBFs Blocks of rank 4 powers 2, 3 and 4 (blocks 1, 2 and 3)

For example, the block 1 consists of MBFs $f_{30}(4)=x_{1} x_{2} \vee$ $x_{3} x_{4}$ and $f_{31}(4)=x_{1} x_{3} \vee x_{1} x_{4} \vee x_{2} x_{3} \vee x_{2} x_{4}$. Here $f_{31}(4)=f_{30}^{-1}(4)$ also can be told that this block is generated by MBFs $f_{30}(4)$. The block 1 has 2 more isomorphic blocks one of which consists of MBFs $f_{32}(4)=x_{1} x_{3} \vee x_{2} x_{4}$ and $f_{33}(4)$, and other of $\operatorname{MBFs} f_{34}(4)=x_{1} x_{4} \vee x_{2} x_{3}$ and $f_{35}(4)$. All blocks, isomorphic to the block 1 (including the block 1), are generated by MBFs $f_{30}(4), f_{32}(4)$ and $f_{34}(4)$. These blocks contain 6 MBFs. The 
block 2 consists of MBFs $f_{36}(4), f_{37}(4)$ and $f_{38}(4)$. This block has 3 isomorphic blocks one of which consists of MBFs $f_{39}(4), f_{40}(4)$ and $f_{41}(4)$, other of MBFs $f_{42}(4), f_{43}(4)$ and $f_{44}(4)$, and the third of MBFs $f_{45}(4), f_{46}(4)$ and $f_{47}(4)$. All blocks isomorphic to the block 2 are generated by MBFs $f_{36}(4)=$ $x_{2} x_{3} \vee x_{2} x_{4} \vee x_{3} x_{4}, f_{39}(4)=x_{1} x_{3} \vee x_{1} x_{4} \vee x_{3} x_{4}, f_{42}(4)=x_{1} x_{2} \vee$ $x_{1} x_{4} \vee x_{2} x_{4}$ and $f_{45}(4)=x_{1} x_{2} \vee x_{1} x_{3} \vee x_{2} x_{3}$. These blocks contain 12 MBFs. The block 3 consists of MBFs $f_{48}(4), f_{49}(4)$, $f_{50}(4)$ and $f_{51}(4)$. This block has 2 isomorphic blocks one of which consists of MBFs $f_{52}(4), f_{53}(4), f_{54}(4)$ and $f_{55}(4)$, and other of MBFs $f_{56}(4), f_{57}(4), f_{58}(4)$ and $f_{59}(4)$. All blocks isomorphic to the block 3 are generated by MBFs МБФ $f_{48}(4)$ $=x_{1} x_{2} \vee x_{2} x_{3} \vee x_{3} x_{4}, f_{52}(4)=x_{1} x_{2} \vee x_{1} x_{3} \vee x_{3} x_{4}$ and $f_{56}(4)=x_{1} x_{2}$ $\vee x_{1} x_{4} \vee x_{2} x_{3}$. These blocks contain 12 MBFs. Thus, is available $3+4+3=10$ blocks, to isomorphic three blocks on fig. 5. These blocks contain $6+12+12=30$ MBFs.

On fig. 6 Two MBFs blocks of rank 4 powers of 6 (a) the block 4 and $b$ ) the block 5) are shown.


Figure 6. MBFs Blocks of rank 4 powers 6 (blocks 4 and 5)

The block 4 has no isomorphic and consists of MBFs $f_{5}(4)$, $f_{4}(4), f_{3}(4), f_{2}(4), f_{0}(4)$ and $f_{1}(4)$. The first 4 MBFs and unit MBFs $f_{1}(4)$ are the maximum MBFs of rank 4 weights 1 . MBFs $f_{4}(4)$ and zero MBFs $f_{0}(4)$ are own disjunctive complement, and MBFs $f_{5}(4)$ and MBFs $f_{1}(4)$ are own conjunctive complement. The block 5 consists of MBFs $f_{6}(4)$ $=x_{1}, f_{7}(4)=x_{2} x_{3} x_{4}, f_{8}(4)=x_{2} \vee x_{3} \vee x_{4}, f_{9}(4)=x_{1} x_{2} x_{3} \vee x_{1} x_{2} x_{4}$ $\vee x_{1} x_{3} x_{4}, f_{10}(4)=x_{1} \vee x_{2} x_{3} \vee x_{2} x_{4} \vee x_{3} x_{4}$ and $f_{11}(4)=x_{1} x_{2} \vee$ $x_{1} x_{3} \vee x_{1} x_{4} \vee x_{2} x_{3} x_{4} . \mathrm{MBFs} f_{6}(4)$ and $f_{10}(4)$ are self-dual. The block 5 has three isomorphic blocks one of which consists of MBFs $f_{12}(4), f_{13}(4), f_{14}(4), f_{15}(4), f_{16}(4)$ and $f_{17}(4)$, other of MBFs $f_{18}(4), f_{19}(4), f_{20}(4), f_{21}(4), f_{22}(4)$ and $f_{23}(4)$, and the third of MBFs $f_{24}(4), f_{25}(4), f_{26}(4), f_{27}(4), f_{28}(4)$ and $f_{29}(4)$. All blocks, isomorphic to the block 5 , are generated by MBFs $f_{6}(4)=x_{1}, f_{12}(4)=x_{2}, f_{18}(4)=x_{3}$ and $f_{24}(4)=x_{4}$. In total there are 5 blocks, isomorphic to blocks on fig. 3. These blocks contain 30 MBFs.

On fig. 7 two MBFs blocks of rank 4 powers 12 (a) the block 6 and b) the block 7) are shown.

Blocks 6 and 7 are similar, but are not isomorphic. The block 6 has 2 isomorphic blocks. All blocks isomorphic to the block 6 , are generated by MBFs $f_{60}(4)=x_{1} x_{2}, f_{72}(4)=x_{1} x_{3}$ and $f_{84}(4)=x_{1} x_{4}$. These blocks contain $36 \mathrm{MBFs}$. The block 7 has 5 isomorphic blocks. All blocks isomorphic to the block 7 , are generated by MBFs $f_{96}(4)=x_{1} x_{2} \vee x_{1} x_{3}, f_{108}(4)=x_{1} x_{2} \vee$ $x_{1} x_{4}, f_{120}(4)=x_{1} x_{2} \vee x_{2} x_{3}, f_{132}(4)=x_{1} x_{2} \vee x_{2} x_{4}, f_{144}(4)=x_{1} x_{3} \vee$ $x_{1} x_{4}$ and $f_{156}(4)=x_{1} x_{3} \vee x_{2} x_{3}$. These blocks contain 72 MBFs. In total there are 9 blocks, isomorphic to blocks on fig. 3 . These blocks contain 108 MBFs.
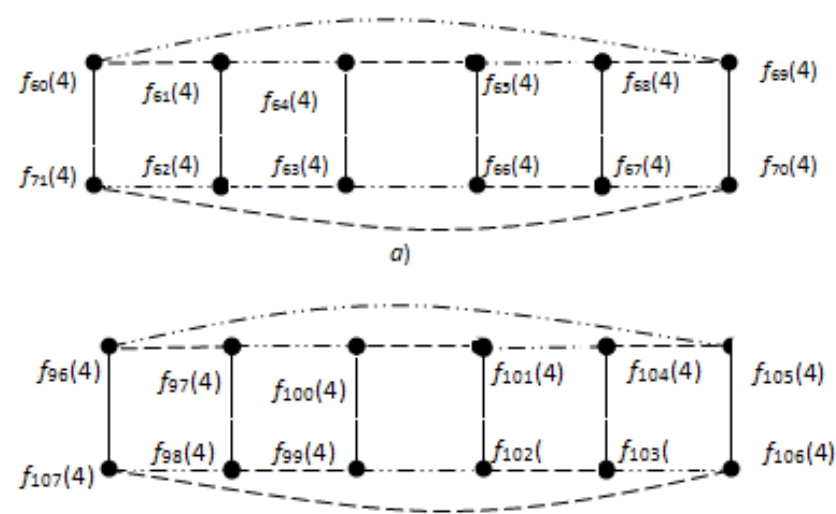

b)

Figure 7. MBFs Blocks of a rank of 4 powers 12 (blocks 6 and 7)

Thus, all 168 MBFs of rank 4 form 24 blocks. Among these blocks only 7 not isomorphic among themselves and 6 are not similar each other. Power block of rank 4 can be one of 5 numbers: 2, 3, 4, 6 and 12 . All MBFs of any block can be obtained from the generator MBFs using two operations, for example, disjunctive complement and a duality. Blocks, isomorphic to some block, it is possible to receive substitution of variables in MBFs entering into this block. Operation of substitution of variables is realised much more simply, than duality operation. Therefore, for complete search of MBFs of the set rank by a method of creation of MBFs blocks it is enough to construct not isomorphic with each other blocks, and the others turn out from them substitution of variables. Already for rank 4 instead of 24 blocks it is enough to construct 7 . With rank growth the relation of all blocks does not isomorphic increases.

For example, we find the Dedekind number to 4 variables. Here there are 7 groups of isomorphic blocks. Of these, by one group of 2, 3 and $4 \mathrm{MBFs}, 2$ groups consist of $6 \mathrm{MBFs}$, and another 2 of $12 \mathrm{MBFs}$. These groups are contained at 3, 4, $3,1,4,3$ and 6 isomorphic blocks. Hence, the Dedekind number $\mathrm{D}(4)=2 * 3+3 * 4+4 * 3+6 * 1+6 * 3+12 * 3+$ $12 * 6=6+12+12+6+18+36+72=168$.

Dedekind number for 5 variables is convenient to count through similar blocks. For the 5 variables, there are 6 groups of similar blocks. Of these, by one group of $4,6,7,14,32$ and $54 \mathrm{MBFs}$, Groups of similar blocks of 4, 6, 32 and 54 MBFs contain on 1 group isomorphic blocks. Group of similar blocks of 7 MBFs includes 7 groups of isomorphic blocks and group of similar blocks of 14 MBFs contains 12 groups of isomorphic blocks. In groups of similar blocks of 4 and $6 \mathrm{MBFs}$ contains 5 and 6 blocks. In the group of 7 similar blocks MBFs contains $1+2 * 5+2 * 10+1 * 20+1$ 
* $30=81$ blocks. In the group of similar blocks of $14 \mathrm{MBFs}$ contains $1 * 10+2 * 15+1 * 20+5 * 30+3 * 60=390$ blocks. In groups of similar blocks of 32 and $54 \mathrm{MBFs}$ contains 30 and 10 blocks. Hence, the fifth Dedekind number $\mathrm{D}(5)=4 * 6+6 * 5+7 * 81+14 * 390+30 * 32+10 * 54=$ $24+30+567+5460+960+540=7581$.

As can be seen from Fig. 3 - 7 all MBFs of similar blocks turn out equally by means of the considered three operations. In some cases, as for the blocks generated MBFs $f_{5}(3)=x_{1}$, $f_{10}(3)=x_{2}$ and $f_{15}(3)=x_{3}$, all of these MBFs blocks obtained from each other by a change of variables. Search of all MBFs of rank $n$ can be arranged as follows. Take MBFs arbitrary rank $n$. by considering the three operations for her construction block. Then taken MBFs arbitrary rank $n$, is not included in the block, and it is also construct block. These actions are repeated until there are MBFs are not included inconstructed blocks. Thus, all MBFs rank $\mathrm{n}$ will be divided into blocks. The advantage of this method is that all MBFs inside the block 3 connected with each other operations and the construction of blocks of the similar blocks can be not build enumeration reduced.

\section{Conclusions}

In summary we will note the following. The method of the analysis and MBFs classification on the basis of creation of MBFs blocks is developed. Application of this method to MBFs analysis with number of variables from 0 to 4 is shown. Application of a method of creation of MBFs blocks becomes simpler, if the number of blocks and number of similar blocks is in advance known. Further it is necessary to study splitting into MBFs blocks of separate ranks, and also to look for regularities of splitting into blocks the general for MBFs of all ranks.

Regularities for the blocks found enough, but they cannot be presented in a single paper. They will be discussed in following papers. In particular, they discovered that using blocks is convenient to assume self-dual and disjunctive self-complementary MBFs. At the same time one cannot say that we will consider all the regularities. Some of them, which relate to the number and size of nonisomorphic to MBFs blocks given rank may still require the efforts of many mathematicians.

\section{REFERENCES}

[1] Dedekind R. Über Zerlegungen von Zahlen durch ihre grössten gemeinsamen Teiler. Festschrift Hoch. Braunschweig u. ges. Werke. II, 1897, 103-148.

[2] Wiedemann, Doug A computation of the eighth Dedekind number. Order 8 (1991), no. 1, 5-6.

[3] Pieter-Jan Hoedt, Parallelizing with MPI in Java to Find the ninth Dedekind Number, http://oeis.org/A000372, 2015.

[4] Ткаченко В.Г. Классификация монотонных булевых функций при синтезе цифровых схем. Наукові праці ОНАЗ ім. О.С. Попова, 2008, № 1, 35 - 43.

[5] Ткаченко В.Г. Перечисление типов монотонных булевых функций при синтезе цифровых схем. Наукові праці ОНАЗ ім. О.С. Попова, 2008, № 2, $54-69$.

[6] А.М.Иваницкий, В.Г. Ткаченко Взаимосвязь между матроидами и монотонными булевыми функциями электрических цепей. Наукові праці ОНАЗ ім. О.С. Попова, 2009, № 1, 18 - 26 .

[7] В.Г. Ткаченко, О.В. Синявский Построение корректирующего кода для криптосистем на основе типов монотонных булевых функций. Наукові праці ОНАЗ ім. О.С. Попова, 2010, № 1, 85 - 92.

[8] Ткаченко В.Г. Взаимосвязь между всеми типами и максимальными типами монотонных булевых функций. Наукові праці ОНАЗ ім. О.С. Попова, 2010, № 2, 60 - 69. 